\title{
Total Knee Arthroplasty for Severe Flexion Contracture in Rheumatoid Arthritis Knees
}

Youn Soo Hwang, MD, Kyu Pill Moon, MD, Kyung Taek Kim, MD, Jin Wan Kim, MD, and Won Seok Park, MD Department of Orthopaedic Surgery, Dong-Eui Medical Center, Busan, Korea

\begin{abstract}
Flexion contracture deformities, as well as severe varus and valgus deformities of the knee joint, accompany osteoarthritis or rheumatoid arthritis (RA). In particular, severe flexion contracture deformity of the knee joint is often found in patients with RA, which renders them nonambulatory. This report describes a 26-year-old female patient diagnosed with RA 10 years ago. She had chronic joint pain, severe flexion contracture, valgus deformity in both knees, and limited range of motion in both knees and became nonambulatory. She underwent a total knee arthroplasty (TKA) and serial casting and physical therapy to restore stable joint movement and correct knee joint deformity. Her pain was successfully relieved, and she was able to walk after surgery. Here, we report the excellent results of TKA in this RA patient with severe flexion contracture of both knees.
\end{abstract}

Keywords: Knee, Rheumatoid arthritis, Arthroplasty, Flexion contracture

Flexion contracture deformities, as well as severe varus and valgus deformities of the knee joint, accompany osteoarthritis (OA) or rheumatoid arthritis (RA). Various factors can cause flexion contracture in arthritic knee joints, such as deformities of the ligament, joint capsule, and bone. Flexion contracture causes severe pain and impairs knee joint function, resulting in loss of ambulation when the angle exceeds $30^{\circ}$ and restriction to daily activities. Various methods are available for the treatment of flexion contracture deformity depending on the severity of contracture. One is total knee replacement, which reportedly relieves pain caused by flexion contracture deformities, stabilizes knee joint movement, and corrects flexion contracture with excellent results ${ }^{1)}$. We performed a total knee arthroplasty (TKA) according to the rule of one-third correction of flexion contracture and conducted serial casting and physical therapy in a patient with a

Received April 11, 2016; Revised (1st) June 13, 2016;

(2nd) August 4, 2016; Accepted August 5, 2016

Correspondence to: Kyu Pill Moon, MD

Department of Orthopaedic Surgery, Dong-Eui Medical Center, 62

Yangjeong-ro, Busanjin-gu, Busan 47227, Korea

Tel: +82-51-850-8937, Fax: +82-51-850-8943

E-mail: moonkp@hanmail.net

This is an Open Access article distributed under the terms of the Creative Commons Attribution Non-Commercial License (http://creativecommons.org/licenses/by-nc/4.0/) which permits unrestricted non-commercial use, distribution, and reproduction in any medium, provided the original work is properly cited. severe $90^{\circ}$ flexion contracture and valgus deformity caused by RA in both knees. Preoperatively, the patient had limited knee joint motion and inability to walk. The procedure restored stable joint movement and corrected knee joint deformity. Her pain was successfully relieved, and she was able to walk after surgery. Herein, we report this case with a literature review.

\section{Case Report}

A 26-year-old female patient with chronic RA visited the hospital because of chronic joint pain in both knees and limited knee joint motion induced by severe flexion contracture and valgus deformity. She had RA diagnosed 10 years ago and had been undergoing conservative treatment. The pain in both knees was aggravated since 5 years ago, and a severe flexion contracture deformity developed gradually, resulting in inability to walk. The range of motion (ROM) of the right and left knees was limited to $85^{\circ}-135^{\circ}$ and $90^{\circ}-130^{\circ}$, respectively, which was accompanied by a valgus deformity. Severe flexion contracture and valgus deformity were observed on the plain radiographs and scanogram (Fig. 1A-C). Both knees had severe joint destruction and deformity caused by RA, which was the indication for TKA. Operation was decided for the left knee first due to the presence of more severe pain and greater flexion contracture deformity than the right knee. Although preoperative application of serial casting and 

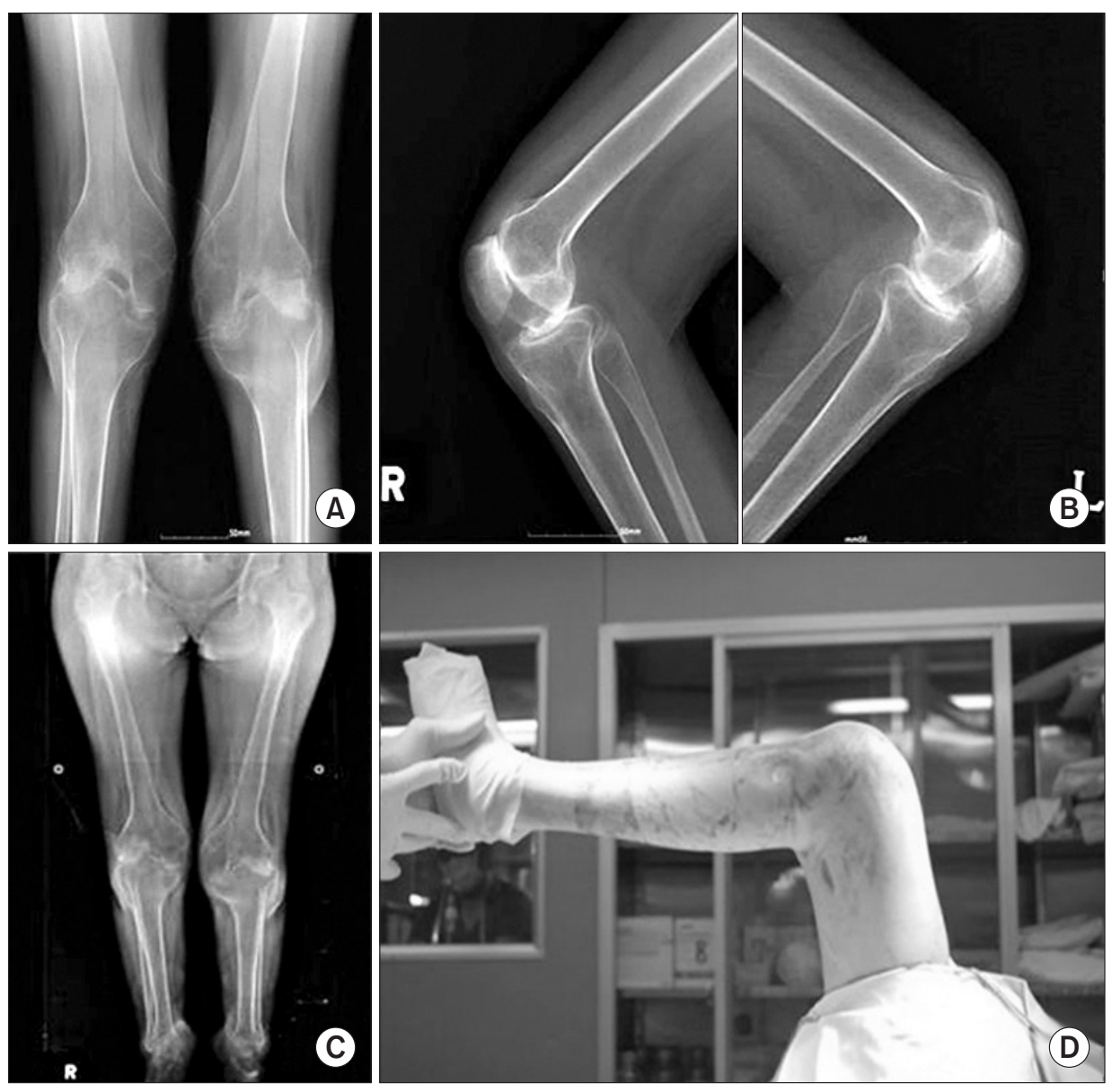

Fig. 1. Preoperative anteroposterior (A) and lateral (B) radiographs of both knees and weight bearing full-length scanogram (C). (D) The preoperative flexion contracture of the left knee was $90^{\circ}$.

physical therapy may be more effective for severe flexion contracture in both knees, we decided to perform TKA and proceed with serial casting and physical therapy postoperatively to correct the remaining flexion contracture. Furthermore, considering the possibility that she might need additional revision knee arthroplasty in the future, as well as her young age, the goals of surgery were to perform a proper soft tissue procedure and minimal osteotomy for one-third correction of the flexion contracture deformity.

Surgery was performed under spinal anesthesia and the $90^{\circ}$ flexion contracture of the left knee measured in the preoperative test was confirmed in surgery (Fig. 1D). Using the general medial parapatellar approach, the hyperplastic synovium was removed. Femoral posterior condyle osteophyte removal was carried out and a proper posterior soft tissue release was performed. Then, additional distal femoral resection of more than $2 \mathrm{~mm}$ was performed to treat the flexion contracture by widening the extension gap. The posterior cruciate ligament (PCL) was resected to balance the flexion/extension gap and a PCL-substituting implant (e.motion; B. Braun-Aesculap, Tuttlingen, Germany) was inserted.
Immediately postoperatively, plain radiography of the left knee was performed: the anteroposterior and lateral views of the left knee joint revealed that an additional extension of approximately $29^{\circ}$ was attained (Fig. 2A). Quadriceps setting exercise was begun immediately postoperatively, and serial casting and physical therapy were performed to correct the remaining flexion contracture deformity. Gradual stretching and correction of the posterior capsular structure of the knee joint was also initiated. Three months after the operation, the left knee pain was considerably relieved and full extension was achieved with $0^{\circ}-100^{\circ}$ of ROM (Fig. 2B). Six months after the operation, the patient underwent a TKA for the right knee using the same method, which was minimal ostectomy for one-third correction of the flexion contracture deformity, using the same implant. Immediately postoperatively, anteroposterior and lateral radiographs of the right knee joint revealed an approximately $17^{\circ}$ of increase in knee extension (Fig. 3). Postoperative treatment was done in the same way as in the opposite side. Three months after the operation, the knee joint pain was considerably relieved and the ROM of the right knee was between $0^{\circ}$ and $100^{\circ}$, indicating restoration of full extension. 

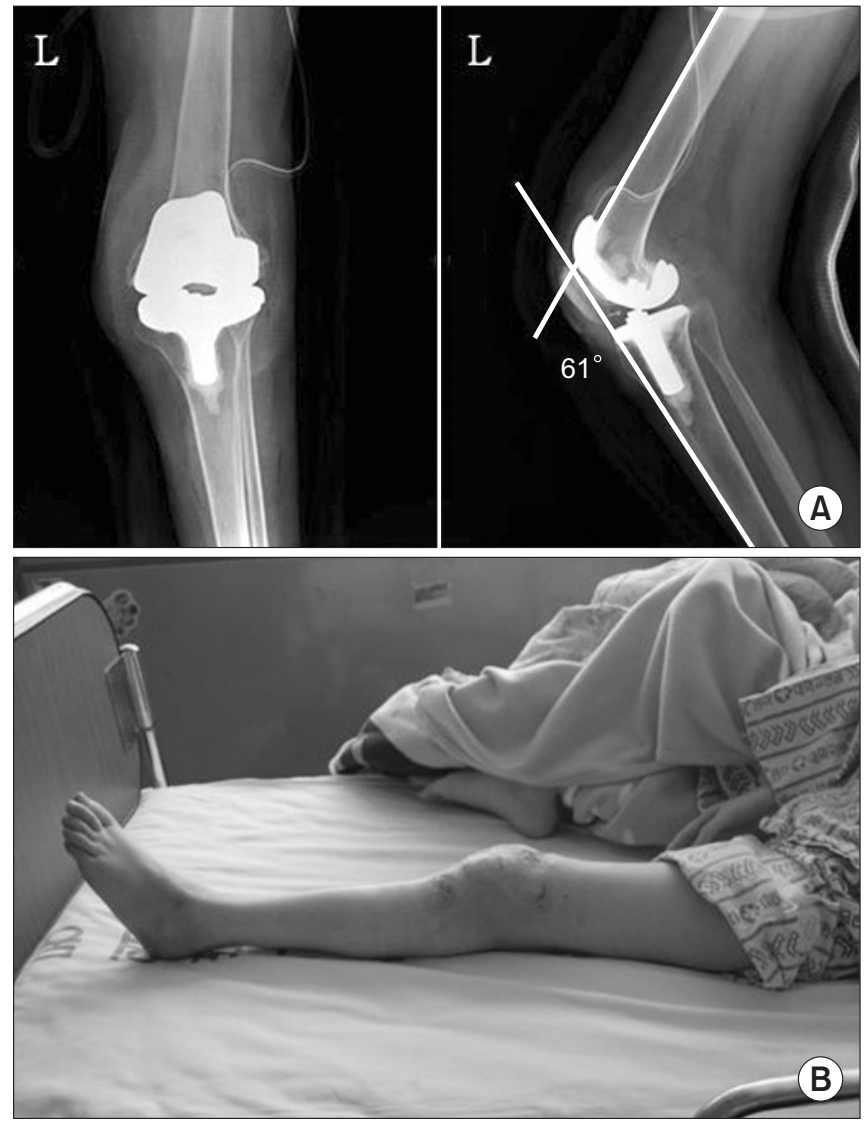

Fig. 2. Anteroposterior and lateral (A) radiographs of the left knee taken after total knee arthroplasty (TKA). (B) Full extenson of the left knee was achieved at 3 months after TKA.
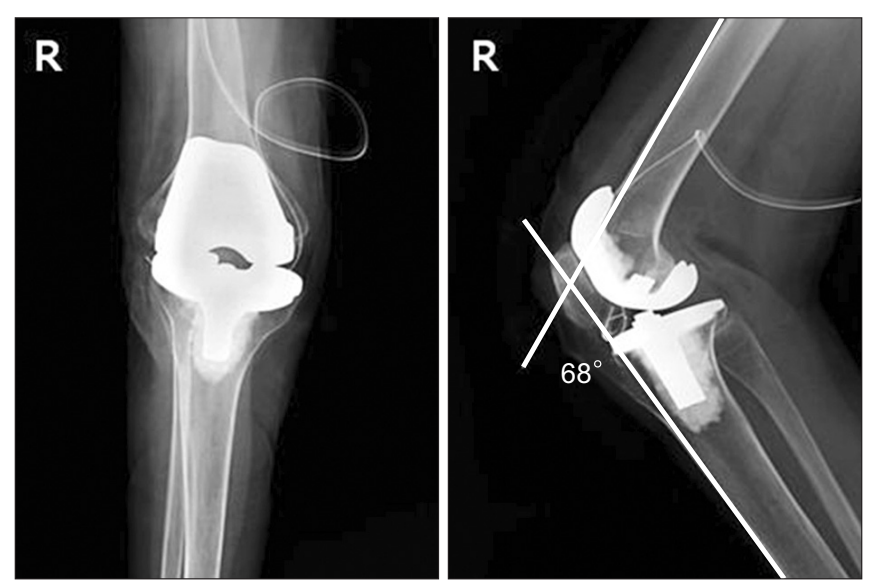

Fig. 3. Anteroposterior and lateral radiographs of the right knee taken after total knee arthroplasty.

During the follow-up period, full extension was achieved in both knees, and the thigh quadriceps muscles and hip and trunk muscles rapidly gained strength by performing standing exercises. Sixth months after the surgery, the patient was able to walk in-
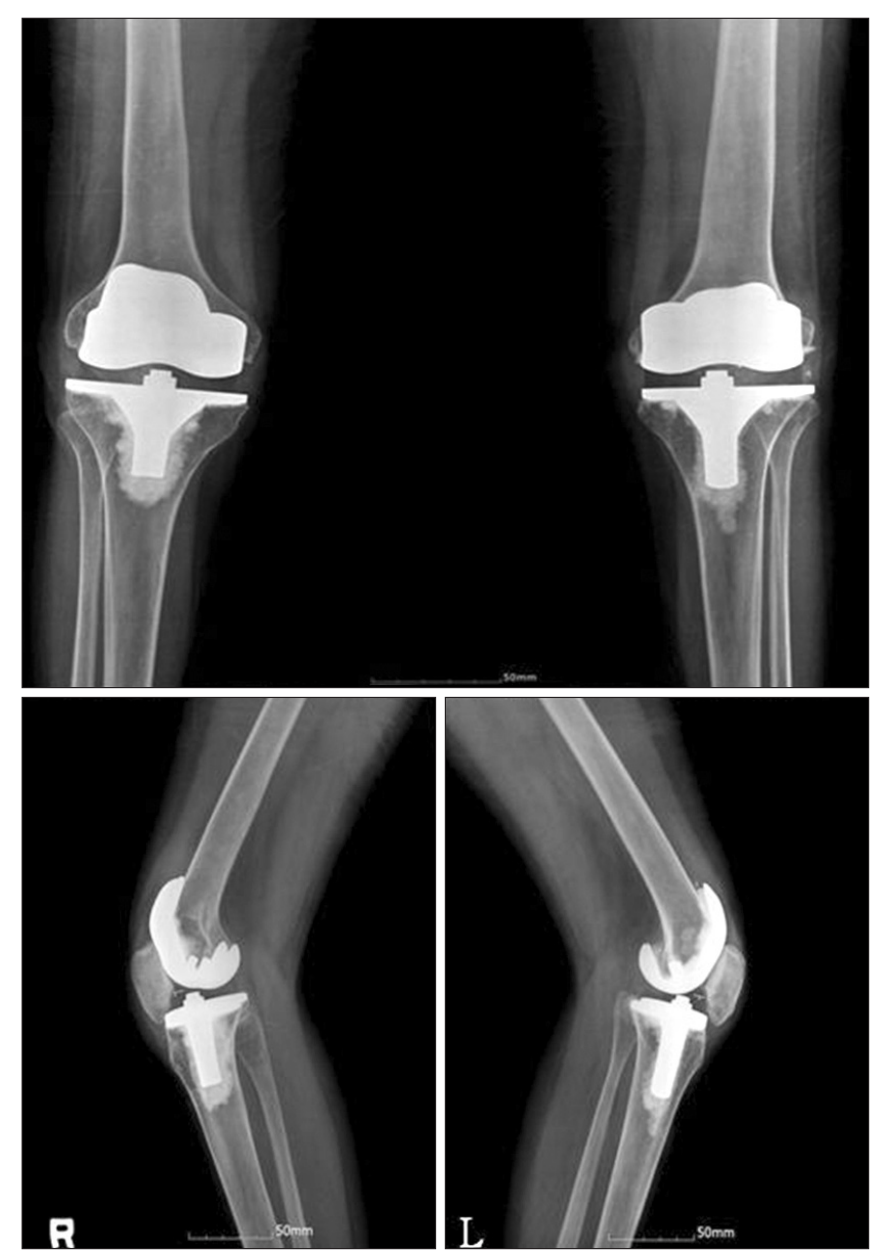

Fig. 4. Anteroposterior and lateral radiographs of both knees at 6 months after right total knee arthroplasty.

dependently without using crutches, and no abnormalities were observed on anteroposterior and lateral radiographs of the knee joints (Fig. 4).

\section{Discussion}

Flexion contracture can result from long-term progression of OA or RA. The mechanism that induces flexion contracture includes inflammation of soft tissues in inflammatory diseases such as RA. Osteophyte formation can be a mechanism in degenerative or traumatic arthritis ${ }^{2}$. Severe flexion contracture of the knee joint is usually accompanied by posterior subluxation of the tibia, valgus deformity, and external rotation of the tibia. These can be partly caused by contracture and traction of the biceps muscle and iliotibial band $d^{3)}$.

In general, attempts are made to improve flexion contracture with splinting, corrective serial casting, skeletal traction, or operative joint mobilization ${ }^{3}$. In addition, depending on the degree 
of deformity, TKA can be a therapeutic option that is reportedly effective for relieving pain caused by flexion contracture, attaining stable knee joint motion, and correcting flexion contracture deformity ${ }^{4)}$.

Various treatment methods of flexion contracture have been used during TKA in accordance with the following deformity angle categories: $<15^{\circ}, 15^{\circ}-45^{\circ}, 45^{\circ}-60^{\circ}$, and $\geq 60^{\circ}$. Treatment methods include osteophyte removal, posterior capsule separation, additional distal femoral resection, tibial posterior slope adjustment, and PCL resection ${ }^{5)}$. Furthermore, $\mathrm{Scott}^{2)}$ in a recent publication described that preoperative manipulation and serial casting should be considered in the case of severe flexion contracture, and a PCL-substituting technique should always be used for flexion contractures of $45^{\circ}-60^{\circ}$. For greater than $60^{\circ}$ of flexion contractures, preoperative manipulation and serial casting are also considered appropriate, and total condylar III stability prosthesis should often be used to resolve flexion gap laxity.

Firestone et al. ${ }^{6}$ reported that serial casting performed 2-4 weeks before operation successfully corrected knee joint flexion contracture of approximately $20^{\circ}$. Laskin and O'Flynn ${ }^{7)}$ recommended preoperative reduction of below $45^{\circ}$ of flexion contracture. $S \mathrm{Cott}^{2}$ in a recent publication suggested that intraoperative flexion contracture correction can be an important guideline that determines final results; however, it was noted that the "rule of one third" should be applied to flexion contracture greater than $40^{\circ}$ identified preoperatively under anesthesia in patients with inflammatory arthritis (e.g., RA). This rule states that intraoperative correction must be limited to one third of the flexion contracture assessed preoperatively under anesthesia. This is because the residual one third will resolve satisfactorily with postoperative physical therapy, sometimes supplemented by serial casting or the use of a dynamic splint.

In this study, intraoperative one-third correction of the flexion contracture was performed without preoperative correction. Serial casting and physical therapy were performed immediately after the operation, and complete knee extension was achieved.

Regarding flexion contracture changes during the postoperative follow-up period, some authors assert that complete intraoperative correction is necessary to correct all flexion contracture deformities ${ }^{6}$, because the remaining knee joint deformity does not improve over time. However, Tanzer and Miller ${ }^{8}$ recommended that complete surgical correction of flexion contracture is not necessary because the remaining flexion contracture would improve in time after TKA. They do not recommend an additional ostectomy for $30^{\circ}$ of flexion contractures, as intraoperative complete correction is not necessary. Ritter and Stringer" reported that complete surgical correction of flexion contracture was not necessary in the case of preoperative flexion contracture greater than $15^{\circ}$, as the flexion contracture would improve considerably within 6 months after surgery.

In this report, we described a 26-year-old female patient with RA diagnosed 10 years ago. When she visited the hospital, she had chronic joint pain in both knees and limited knee joint motion induced by severe flexion contracture and valgus deformity. Since 5 years before presentation to our hospital, severe flexion contracture deformity had developed gradually, making her unable to stand and walk. So we aimed to correct the severe flexion contracture deformity to restore her normal walking condition by performing TKA followed by serial casting and physical therapy. The patient's knee joint motion was restored through knee deformity correction. The treatment successfully relieved her pain, and she was able to walk again. We did not perform flexion contracture correction before surgery, considering the previous reports suggesting that in patients with RA combined with severe flexion contracture of the knee joint, the remaining flexion contracture after surgery can be effectively treated with serial casting and physical therapy. Furthermore, considering the possibility that the patient might need additional revision knee arthroplasty in the future and her young age, TKA was performed using general PCL-substituting implants. After correction of one third of the flexion contracture deformity through TKA, the patient underwent serial casting and physical therapy to correct the remaining flexion contracture. The treatment results were successful, and she was able to walk again 6 months after the surgery. Further studies are needed on the corrective methods for severe flexion contractures greater than $60^{\circ}$.

\section{Conflict of Interest}

No potential conflict of interest relevant to this article was reported.

\section{References}

1. Chung HK, Choi CH, Choi YJ, Kim JH. Total knee replacement arthroplasty in severe flexion contracuture. J Korean Orthop Assoc. 1997;32:1039-46.

2. Scott RD. Total knee arthroplasty. 2nd ed. Philadelphia, PA: Elsevier; 2014. p75-8.

3. Tateishi H. Contracture of the knee joint. J Joint Surg. 1985; 4:361-5.

4. Laskin RW, Beksac B. Assess and achieve maximal exten- 
sion. In: Bellemans J, Ries MD, Victor J, eds. Total knee arthroplasty: a guide to get better performance. Berlin: Springer; 2005. p194-7.

5. Scuderi GR, Kochhar T. Management of flexion contracture in total knee arthroplasty. J Arthroplasty. 2007;22(4 Suppl 1): 20-4.

6. Firestone TP, Krackow KA, Davis JD 4th, Teeny SM, Hungerford DS. The management of fixed flexion contractures during total knee arthroplasty. Clin Orthop Relat Res. 1992; (284):221-7.
7. Laskin RS, O'Flynn HM. The Insall Award: total knee replacement with posterior cruciate ligament retention in rheumatoid arthritis: problems and complications. Clin Orthop Relat Res. 1997;(345):24-8.

8. Tanzer M, Miller J. The natural history of flexion contracture in total knee arthroplasty: a prospective study. Clin Orthop Relat Res. 1989;(248):129-34.

9. Ritter MA, Stringer EA. Predictive range of motion after total knee replacement. Clin Orthop Relat Res. 1979;(143):115-9. 